\title{
Koszulness, Krull dimension, and other properties of graph-related algebras
}

\author{
Alexandru Constantinescu • Matteo Varbaro
}

Received: 25 September 2009 / Accepted: 11 January 2011 / Published online: 4 February 2011

(C) Springer Science+Business Media, LLC 2011

\begin{abstract}
The algebra of basic covers of a graph $G$, denoted by $\bar{A}(G)$, was introduced by Herzog as a suitable quotient of the vertex cover algebra. In this paper we compute the Krull dimension of $\bar{A}(G)$ in terms of the combinatorics of $G$. As a consequence, we get new upper bounds on the arithmetical rank of monomial ideals of pure codimension 2. Furthermore, we show that if the graph is bipartite, then $\bar{A}(G)$ is a homogeneous algebra with straightening laws, and thus it is Koszul. Finally, we characterize the Cohen-Macaulay property and the Castelnuovo-Mumford regularity of the edge ideal of a certain class of graphs.
\end{abstract}

Keywords Vertex covers of graphs · Cover ideal · Edge ideal · Fiber cone - Koszul · Straightening laws · Krull dimension · Arithmetical rank · Cohen-Macaulay · Castelnuovo-Mumford regularity

\section{Introduction}

Due to their relation with the resolution of singularities of schemes, blowup algebras are an important subject in both Commutative Algebra and Algebraic Geometry. On the other hand, the vertex covers of a graph are important objects in Graph Theory, having many practical applications. In this paper we are going to study blowup algebras related to graphs, merging the above topics. In fact, these algebras have an interpretation in terms of the vertex covers, more precisely the $k$-covers, of the graph.

\footnotetext{
A. Constantinescu $(\bowtie) \cdot$ M. Varbaro

Dipartimento di Matematica, Università degli Studi di Genova, Via Dodecaneso 35, Genova 16146, Italy

e-mail: a.constantinescu@unibas.ch

M. Varbaro

e-mail: varbaro@dima.unige.it
} 
Many ring-theoretic properties are thus described in terms of the combinatorics of the graph.

Given a graph $G$ on $n$ vertices, its cover ideal is the ideal $J(G)=\bigcap\left(x_{i}, x_{j}\right) \subseteq$ $K\left[x_{1}, \ldots, x_{n}\right]$, where the intersection runs over the edges of $G$. The symbolic Rees algebra of this ideal is also known as the vertex cover algebra of $G$. In their paper [13] Herzog, Hibi, and Trung have studied this algebra in the more general context of hypergraphs. In the present paper we study the symbolic fiber cone of $J(G)$, denoted by $\bar{A}(G)$. There are three main results:

(a) A combinatorial characterization of the Krull dimension of $\bar{A}(G)$ (Theorem 2.8). This problem was raised by Herzog in 2008. As a nice consequence, we give an upper bound for the number of equations defining up to radical a monomial ideal of codimension 2, refining a result of Lyubeznik obtained in [20].

(b) The Koszul property of $\bar{A}(G)$ for a bipartite graph $G$ (Theorem 3.4). This problem was suggested by Herzog too, during an informal conversation at Oberwolfach in 2009. Actually we prove more: If $G$ is bipartite, then $\bar{A}(G)$ has a natural structure of homogeneous algebra with straightening laws. From the arising poset we give many examples of bipartite graphs for which $\bar{A}(G)$ has or does not have certain ring-theoretic properties.

(c) A combinatorial criterion for the Cohen-Macaulayness of edge ideals of graphs satisfying the weak square condition (Theorem 4.7). Characterizing the graphs for which the edge ideal is Cohen-Macaulay is a wide open question and a very studied problem. Our result generalizes a theorem by Herzog and Hibi obtained in [12], where they characterize the bipartite graphs for which the edge ideal is Cohen-Macaulay.

Let us describe how the paper is organized.

In the first section we recall the definition and basic properties of the symbolic fiber cone of the cover ideal of a graph, namely $\bar{A}(G)$. In Sect. 2 we compute, in terms of the combinatorics of the graph, the dimension of $\bar{A}(G)$. The combinatorial invariant that we introduce is called the ordered matching number. It turns out that it has a lower bound given by the paired-domination number and an upper bound given by the matching number of the graph. When the base field is infinite, the dimension of $\bar{A}(G)$ is an upper bound for the arithmetical rank of $J(G)$ localized at the maximal irrelevant ideal, and so we get interesting upper bounds for the arithmetical rank of a monomial ideal of pure codimension 2 after having localized at the maximal irrelevant ideal, thus improving a result of [20].

In the third section of the paper we prove that for a bipartite graph $G$, the algebra $\bar{A}(G)$ is Koszul. The Koszul property follows from the homogeneous ASL structure which we can give to $\bar{A}(G)$. In a joint paper with Benedetti [1], we gave for a bipartite graph a combinatorial condition equivalent to $\bar{A}(G)$ being a domain. This combinatorial property is called weak square condition (WSC). The ASL structure provides in the bipartite case another equivalent condition: $\bar{A}(G)$ is a domain if and only if $\bar{A}(G)$ is a Hibi ring. Using this structure and a result of Hibi from [14], we are able to characterize for bipartite graphs the Gorenstein domains. The nonintegral case turns out to be more complicated. However, from the description of the poset on which $\bar{A}(G)$ is an ASL we can deduce some nice consequences. For instance, we can 
produce many examples of bipartite graphs such that $\bar{A}(G)$ is not Cohen-Macaulay, using results of Kalkbrener and Sturmfels [15] and of the second author [25]. With some additional assumption on the combinatorics of the graph, we can prove that $\bar{A}(G)$ is Cohen-Macaulay if and only if it is equidimensional.

In the fourth and last section we focus our attention on the edge ideal of the graph, namely $I(G)=\left(x_{i} x_{j}:\{i, j\}\right.$ is an edge of $\left.G\right) \subseteq S=K\left[x_{1}, \ldots, x_{n}\right]$. Two problems that have recently caught the attention of many authors (see, for instance, $[8,9$, $12,16,17,26])$ are the characterization, in terms of the combinatorics of $G$, of the Cohen-Macaulay property and the Castelnuovo-Mumford regularity of $S / I(G)$. Our approach is to restrict the problem to a subgraph $\pi(G)$ of $G$ which maintains some useful properties of the edge ideal. This graph is constructed by passing through another graph, namely $G^{0-1}$, introduced by Benedetti and the second author in [2]. Using this tool, we are able to extend a result of [12] regarding the Cohen-Macaulay property and a result of Kummini from [17] regarding the Castelnuovo-Mumford regularity.

\section{Terminology and preliminaries}

For the convenience of the reader, we include in this short section the standard terminology and the basic facts about the algebra of basic covers of a graph.

For a natural number $n \geq 1$, we denote by $[n]$ the set $\{1, \ldots, n\}$. By a graph $G$ on $[n]$ we understand a graph with $n$ vertices without loops or multiple edges. If we do not specify otherwise, we also assume that a graph has no isolated points. We denote by $V(G)$ (respectively $E(G)$ ) the vertex set (respectively the edge set) of $G$. From now on $G$ will always denote a graph on $[n]$, and we will write, when it does not raise confusion, just $V$ for $V(G)$ and $E$ for $E(G)$. A subset $V^{\prime} \subseteq V$ is called a vertex cover of $G$ if for any $e \in E$, we have $e \cap V^{\prime} \neq \emptyset$. A vertex cover $V^{\prime}$ is called minimal if no proper subset of $V^{\prime}$ is again a vertex cover. More generally, a nonzero function $\alpha: V(G) \rightarrow \mathbb{N}$, is a $k$-cover of $G(k \in \mathbb{N})$ if $\alpha(i)+\alpha(j) \geq k$ whenever $\{i, j\} \in E(G)$. A $k$-cover $\alpha$ is decomposable if $\alpha=\beta+\gamma$, where $\beta$ is an $h$-cover, and $\gamma$ is a $(k-h)$ cover; $\alpha$ is indecomposable if it is not decomposable. A $k$-cover $\alpha$ is called basic if it is not decomposable as a 0 -cover plus a $k$-cover (equivalently if no function $\beta<\alpha$ is a $k$-cover). Notice the correspondence between basic 1 -covers and minimal vertex covers.

Throughout the paper, $K$ will be a field, $S=K\left[x_{1}, \ldots, x_{n}\right]$ will denote the polynomial ring with $n$ variables over $K$, and $\mathfrak{m}=\left(x_{1}, \ldots, x_{n}\right)$ will be the irrelevant maximal ideal of $S$. The edge ideal of $G$, denoted by $I(G)$, is the square-free monomial ideal of $S$

$$
I(G)=\left(x_{i} x_{j}:\{i, j\} \in E(G)\right) \subseteq S .
$$

A graph $G$ is called Cohen-Macaulay over $K$ if $S / I(G)$ is a Cohen-Macaulay ring. A graph is called just Cohen-Macaulay if it is Cohen-Macaulay over any field (equivalently over $\mathbb{Z}$ ). The cover ideal of $G$ is the Alexander dual of the edge ideal, and we denote it by $J(G)$. So

$$
J(G)=\bigcap_{\{i, j\} \in E(G)}\left(x_{i}, x_{j}\right)
$$


As said in the introduction, in this paper we study the symbolic fiber cone of $J(G)$. To introduce it, we recall the definition of the symbolic Rees algebra of an ideal $I \subseteq S$ :

$$
R(I)_{s}=\bigoplus_{k \geq 0} I^{(k)} t^{k} \subseteq S[t]
$$

where $I^{(k)}$ denotes the $k$ th symbolic power of $I$, i.e., $I^{(k)}=\left(I^{k} S_{W}\right) \cap S$, where $W$ is the complement in $S$ of the union of the associated primes of $I$, and $S_{W}$ denotes the localization of $S$ at the multiplicative system $W$. If $I$ is a square-free monomial ideal, then $I^{(k)}$ is just the intersection of the (ordinary) $k$-powers of the minimal prime ideals of $I$. Therefore,

$$
(J(G))^{(k)}=\bigcap_{\{i, j\} \in E(G)}\left(x_{i}, x_{j}\right)^{k} .
$$

The symbolic fiber cone of $I$ is $R(I)_{S} / \mathfrak{m} R(I)_{s}$. We will denote by $\bar{A}(G)$ the symbolic fiber cone of $J(G)$.

There is a more combinatorial way to construct $\bar{A}(G)$, given by the relation between basic covers and $J(G)$ :

$$
J(G)^{(k)}=\left(x_{1}^{\alpha(1)} \cdots x_{n}^{\alpha(n)}: \alpha \text { is a basic } k \text {-cover }\right) .
$$

Thus $R(J(G))_{s}=K\left[x_{1}^{\alpha(1)} \cdots x_{n}^{\alpha(n)} t^{k}: \alpha\right.$ is a $k$-cover $] \subseteq S[t]$. For more details on this interpretation of these algebras, see [13], in which this symbolic Rees algebra is denoted by $A(G)$. The authors of that paper proved many properties of $A(G)$. First of all, they noticed that $A(G)$ is a finitely generated $K$-algebra, since it is generated in degree less than or equal to 2. Moreover $A(G)$ is a standard graded $S$-algebra if and only if $G$ is bipartite. They also proved that $A(G)$ is always a Gorenstein normal domain.

Since $\bar{A}(G)=A(G) / \mathfrak{m} A(G)$, we have that

$$
\bar{A}(G)=K \oplus\left(\bigoplus_{k \geq 1}\left\langle x_{1}^{\alpha(1)} \cdots x_{n}^{\alpha(n)} t^{k}: \alpha \text { is a basic } k \text {-cover }\right\rangle\right),
$$

where the multiplication table is given by

$$
\begin{aligned}
& x_{1}^{\alpha(1)} \cdots x_{n}^{\alpha(n)} t^{k} \cdot x_{1}^{\beta(1)} \cdots x_{n}^{\beta(n)} t^{h} \\
& \quad= \begin{cases}x_{1}^{\gamma(1)} \cdots x_{n}^{\gamma(n)} t^{h+k} & \text { if } \gamma=\alpha+\beta \text { is a basic }(h+k) \text {-cover, } \\
0 & \text { otherwise. }\end{cases}
\end{aligned}
$$

With the above presentation it is clear that the Hilbert function of $\bar{A}(G)$ counts the basic $k$-covers of $G$, i.e.,

$$
\mathrm{HF}_{\bar{A}(G)}(k)=\operatorname{dim}_{K}\left(\bar{A}(G)_{k}\right)=\mid\{\text { basic } k \text {-covers of } G\} \mid .
$$

It turns out that the number of basic $2 h$-covers of a graph grows as a polynomial in $h$ of degree $\operatorname{dim} \bar{A}(G)-1$, namely the Hilbert polynomial $\mathrm{HP}_{\bar{A}(G)^{(2)}}$ of the second 
Veronese subring of $\bar{A}(G)$, which is standard graded (see Remark 2.6). This simple fact will be crucial in the characterization of the Krull dimension of $\bar{A}(G)$ in terms of $G$.

From the above discussion it follows that $\bar{A}(G)$ is a standard graded $K$-algebra (equivalently it is the ordinary fiber cone of $J(G)$ ) if and only if $G$ is bipartite. The graphs for which $\bar{A}(G)$ is a domain have been characterized in [1] in the bipartite case and in [2] in general. Moreover, if $\bar{A}(G)$ is a domain, then it is Cohen-Macaulay, but it may be non-Gorenstein. When $G$ is bipartite, even if $\bar{A}(G)$ is not a domain, the projective scheme defined by $\bar{A}(G)$ is connected, but not necessarily equidimensional, and therefore it may be non-Cohen-Macaulay (for more details, see [1]).

\section{The Krull dimension of $\bar{A}(G)$}

In this section we will introduce the notion of ordered matching number. This notion extends the one of graphical dimension of a bipartite graph introduced in [1]. In [1] it was conjectured that for a bipartite graph, the Krull dimension of $\bar{A}(G)$ is equal to the graphical dimension of $G$, which as we will see in a moment is equal to one plus the ordered matching number of $G$. We will prove that this is true not only in the case of bipartite graphs, but for any graph $G$. As consequences of this result, we are able to give interesting upper bounds for the arithmetical rank of monomial ideals of pure codimension 2 in the localization $S_{\mathfrak{m}}$, refining in this case an upper bound given in [20].

Given a graph $G$, we recall that a set $M \subseteq E(G)=E$ of edges is a matching of $G$ if any two distinct edges of $M$ have empty intersection. A matching is called maximal if it has maximal cardinality among all matchings of $G$. The matching number of $G$, denoted by $v(G)$, is the cardinality of a maximal matching of $G$. A matching $M$ is called perfect if every vertex in $V$ belongs to an edge in $M$. A set of vertices $V^{\prime} \subseteq V(G)=V$ is called independent if $\{v, w\} \notin E$ for any $v, w \in V^{\prime}$. Let $M=$ $\left\{\left\{a_{i}, b_{i}\right\}: i=1, \ldots, r\right\}$ be a nonempty matching of $G$. We will say that $M$ is an ordered matching if:

- $\left\{a_{1}, \ldots, a_{r}\right\}=A \subseteq V$ is a set of independent vertices,

- $\left\{a_{i}, b_{j}\right\} \in E$ implies $i \leq j$.

In this case we will call $A$ a free parameter set and $B=\left\{b_{1}, \ldots, b_{r}\right\} \subseteq V$ a partner set of $A$.

Definition 2.1 Let $G$ be a graph. We define the ordered matching number of $G$ as:

$$
v_{o}(G):=\max \{|M|: M \subseteq E \text { is an ordered matching }\} .
$$

\section{Remark 2.2}

(1) Being an ordered matching depends on the labeling of the vertices in both $A$ and $B$.

(2) In the case of bipartite graphs it is not difficult to verify that the notion of ordered matching number is equivalent to that of graphical dimension given in [1]. In 
fact, using the notation of [1], we have $v_{o}(G)=\operatorname{gdim}(G)-1$ for each bipartite graph $G$.

(3) In general, $B$ is not necessarily a set of independent vertices.

The ordered matching number of a graph is not always easy to compute, and we were not able to express it in terms of classical invariants of graphs in general. In the following example we will see that it does not depend on the local degree of the vertices. By the local degree of a vertex we understand the number of edges incident to that vertex.

Example 2.3 Let $G$ and $G^{\prime}$ be the bipartite graphs represented below. If $V(G)=$ $A \cup B$ and $V\left(G^{\prime}\right)=A^{\prime} \cup B^{\prime}$, it turns out that all four sets have two vertices of local degree 2 and two vertices of local degree 3 . However, we have $v_{o}(G)=2$ and $v_{o}\left(G^{\prime}\right)=3$.
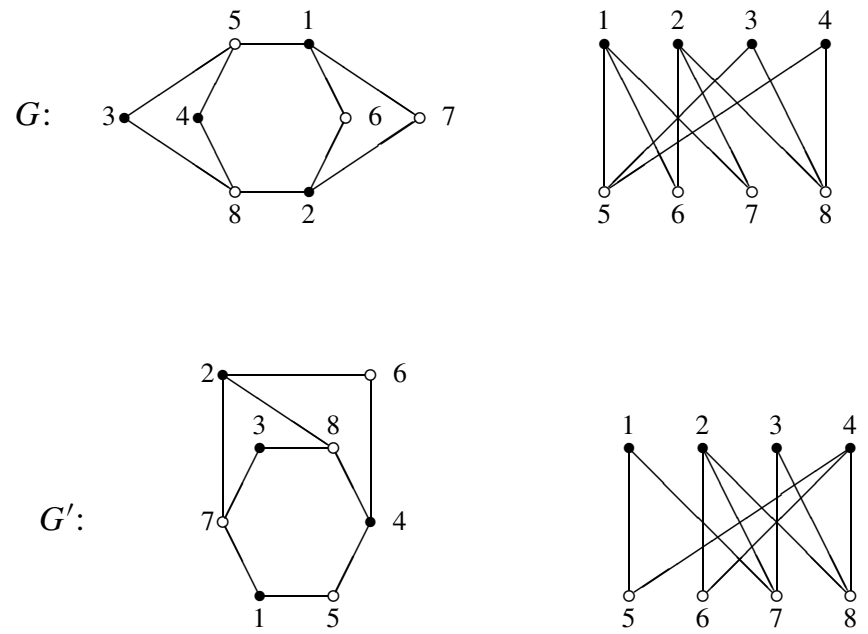

For $G$, an ordered matching of maximal cardinality is $\{\{1,5\},\{2,6\}\}$. For $G^{\prime}$, we have that $\{\{1,5\},\{2,6\},\{3,7\}\}$ is an ordered matching of maximal cardinality. In general these ordered matchings are not unique. For instance, another ordered matching of cardinality 2 for $G$ is $\{\{2,6\},\{3,8\}\}$.

A subset $V^{\prime} \subseteq V$ is a point cover of $G$ if for each $v \in V \backslash V^{\prime}$, there exists a vertex $w \in V^{\prime}$ such that $\{v, w\} \in E$. Notice that a vertex cover is a point cover, but the converse is false. An easy example is given by the triangle $G=K_{3}$ : any vertex of $G$ is a point cover, but not a vertex cover.

Remark 2.4 We recall that a set $S \subseteq V$ is called a paired-dominating set of $G$ if $S$ is a point cover of $G$ and if the subgraph induced by $S$ has at least one perfect matching. The minimum cardinality of a paired-dominating set is called the paired-domination 
number of $G$ and is denoted by $\gamma_{\mathrm{P}}(G)$. The following inequalities hold:

$$
\frac{\gamma_{\mathrm{P}}(G)}{2} \leq v_{o}(G) \leq v(G)
$$

The second inequality is straightforward from the definition. To see the first one, suppose that $A=\left\{a_{1}, \ldots, a_{r}\right\}$ is a free parameter set with partner set $B=\left\{b_{1}, \ldots, b_{r}\right\}$. If $\gamma_{\mathrm{P}}(G)>2 r$, then there is a vertex $v$ in $V \backslash(A \cup B)$ adjacent to none of the vertices of $A \cup B$. Choose a vertex $w$ adjacent to $v$, and set $a_{r+1}=v, b_{r+1}=w$. It turns out that $\left\{a_{1}, \ldots, a_{r}, a_{r+1}\right\}$ is a free parameter set with partner set $\left\{b_{1}, \ldots, b_{r}, b_{r+1}\right\}$.

Example 2.5 In this example we will see that the ordered matching number may reach both the upper and lower bound given in the previous remark. The thick lines in the pictures on the left represent the edges of a perfect matching of a minimal paired dominating set.
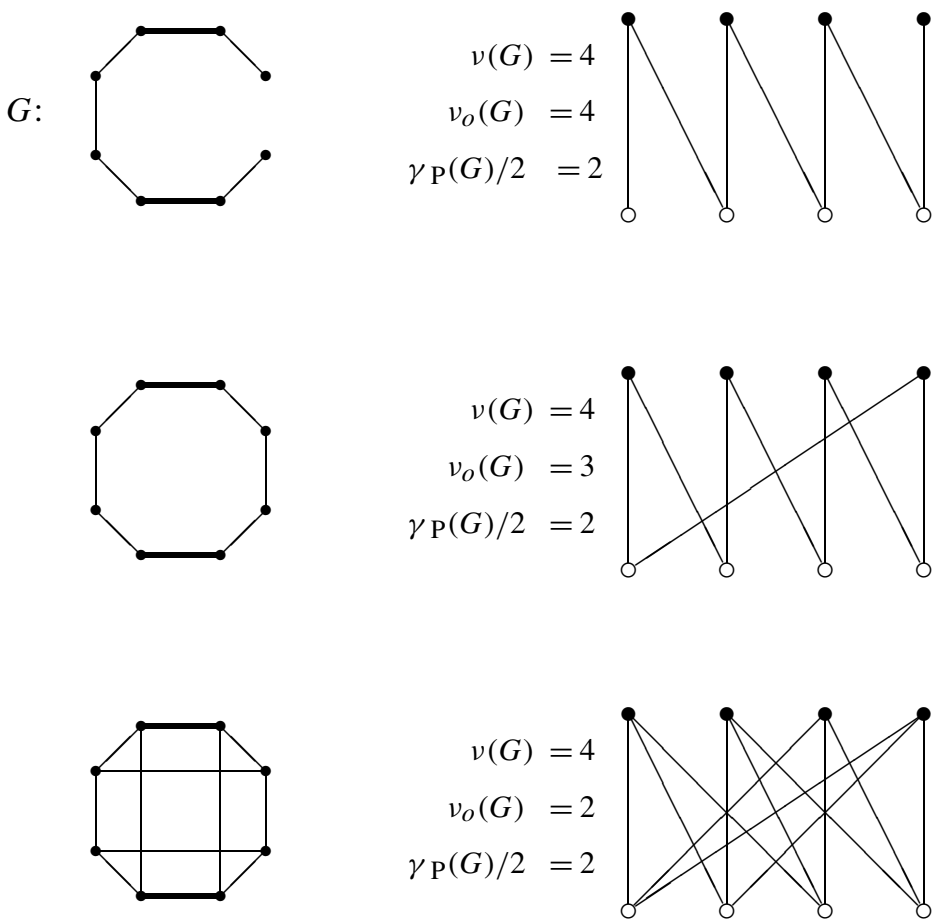

In spite of the examples above, the ordered matching number is easy to compute at least for trees. In this case, $v_{o}(G)=v(G)$ (Proposition 2.10), and there are many algorithms that compute the matching number of a bipartite graph.

To prove the main result of this section, the following remark and lemma are crucial. 
Remark 2.6 There exists a polynomial $P \in \mathbb{Q}[t]$ of degree $\operatorname{dim}(\bar{A}(G))-1$ such that, for $h \gg 0$,

$$
P(h)=\mid\{\text { basic } 2 h \text {-covers of } G\} \mid \text {. }
$$

To see this, consider the second Veronese subring of $\bar{A}(G)$, namely $\bar{A}(G)^{(2)}=$ $\bigoplus_{h>0} \bar{A}(G)_{2 h}$. By [13, Theorem 5.1.a] we have that $\bar{A}(G)^{(2)}$ is a standard graded $K$-algebra. So it has a Hilbert polynomial, denoted by $\operatorname{HP}_{\bar{A}(G)^{(2)}}$, such that $\operatorname{HP}_{\bar{A}(G)^{(2)}}(h)=\operatorname{dim}_{K}\left(\bar{A}(G)_{2 h}\right)$ for $h \gg 0$. Notice that $\bar{A}(G)$ is a finite $\bar{A}(G)^{(2)}$ module, so $\operatorname{dim}(\bar{A}(G))=\operatorname{dim}\left(\bar{A}(G)^{(2)}\right)$, which is the degree of $\mathrm{HP}_{\bar{A}(G)^{(2)}}$ minus 1. So it is enough to take $P=\operatorname{HP}_{\bar{A}(G)^{(2)}}$.

Lemma 2.7 Let $G$ be a graph, $k>0$ a natural number, and $\alpha$ a basic $k$-cover of $G$. Denote by $A_{k / 2}:=\{v \in V: \alpha(v) \leq k / 2\}$.

(a) The set $A_{k / 2}$ is a point cover of $G$, and $\alpha$ is uniquely determined by the values it takes on the vertices in $A_{k / 2}$.

(b) Suppose that $\operatorname{dim}(\bar{A}(G))>s$. Then there exist $k>0$ and a basic $k$-cover $\alpha$ such that $\left|\left\{\alpha(v): v \in A_{k / 2}\right\}\right| \geq s$.

Proof (a) Denote $W=V \backslash A_{k / 2}=\{w \in V: \alpha(w)>k / 2\}$. As $\alpha$ is basic, for each vertex $w \in W$, there exists a vertex $v$ such that $\{w, v\} \in E$ and $\alpha(w)+\alpha(v)=k$. As $\alpha(w)>k / 2$, we must have that $\alpha(v)<k / 2$. So the set $A_{k / 2}$ is a point cover of $G$. It is easy to see that the only possible choice to extend $\alpha$ on the set $W$ is

$$
\alpha(w)=\max \left\{k-\alpha(v):\{v, w\} \in E, \text { and } v \in A_{k / 2}\right\} .
$$

As $A_{k / 2}$ is a point cover, the set we are considering is not empty for any $w \in W$. In order to obtain a $k$-cover, the value of $\alpha(w)$ has to be less or equal to the right-hand side of the above equality. The fact that $\alpha$ has to be also basic implies that the equality has to hold.

(b) Suppose that there are no $k$ and $\alpha$ as we claim. Then, for every $k \geq 0$, there is a function

$\{$ basic $k$-covers of $G\} \longrightarrow\left\{\left(a_{1}, \ldots, a_{n}\right): 0 \leq a_{i} \leq k / 2\right.$ and $\left.\left|\left\{a_{1}, \ldots, a_{n}\right\}\right|<s\right\}$,

given by associating to each basic $k$-cover $\alpha$, a vector which has the same values as $\alpha$ on $A_{k / 2}$ and is 0 in all the other positions. Point (a) guarantees that this is actually an injection. It is not difficult to see that the cardinality of the set on the right-hand side is equal to $C \cdot k^{s-1}$, where $C$ is a constant depending on $n$ and $s$. Therefore Remark 2.6 implies $\operatorname{dim}(\bar{A}(G)) \leq s$, a contradiction.

Now we can prove the main result of this section.

Theorem 2.8 Let $\bar{A}(G)$ be the symbolic fiber cone of the cover ideal of a graph $G$. Then

$$
\operatorname{dim}(\bar{A}(G))=v_{o}(G)+1 .
$$


Proof We will first prove that $\operatorname{dim}(\bar{A}(G)) \geq v_{o}(G)+1$. By Remark 2.6 we have to show that $\mid\{$ basic $2 h$-covers of $G\} \mid$ grows as a polynomial in $h$ of degree at least $v_{o}(G)$.

Let $M=\left\{\left\{a_{i}, b_{i}\right\}: i=1, \ldots, r\right\}$ be an ordered matching of maximal cardinality for $G$. Denote by $A=\left\{a_{1}, \ldots, a_{r}\right\}$ the free parameter set and by $B=\left\{b_{1}, \ldots, b_{r}\right\}$ the partner set of $A$. Furthermore set $X=A \cup B$. So $v_{o}(G)=r$. Let $k>2 r$ be an even natural number. We will construct a basic $k$-cover of $G$ for every decreasing sequence of numbers:

$$
\frac{k}{2} \geq i_{1}>i_{2}>\cdots>i_{r} \geq 0
$$

As the number of decreasing sequences as above is $\left(\begin{array}{c}k / 2+1 \\ r\end{array}\right)$, this will imply that the degree of $\operatorname{HP}_{\bar{A}(G)^{(2)}}$ is at least $r$, so also that $\operatorname{dim}(\bar{A}(G)) \geq v_{o}(G)+1$. For a decreasing sequence as above and for all $j \in\{1, \ldots, r\}$, we define:

$$
\begin{aligned}
& \alpha\left(a_{j}\right)=i_{j}, \\
& \alpha\left(b_{j}\right)=k-i_{j} .
\end{aligned}
$$

As $G$ is connected, if $V \backslash X \neq \emptyset$, there exists a vertex $v \in V \backslash X$ such that there exists at least one edge between $v$ and $X$. We define

$$
\alpha(v)=\max \{k-\alpha(w): w \in X \text { and }\{v, w\} \in E\},
$$

append $v$ to $X$, and continue in the same way until $\alpha$ is defined for all vertices of $G$. It is easy to see that by construction, for each edge $\{v, w\}$ with $v \notin X$ or $w \notin X$ (or both), we have $\alpha(v)+\alpha(w) \geq k$ and that for each vertex $v \notin X$, there exists another vertex $v^{\prime}$ such that $\alpha(v)+\alpha\left(v^{\prime}\right)=k$. So to check that we defined a basic $k$-cover, we need to focus on the vertices in $X$. Let $\{v, w\}$ be an edge with $v, w \in X$. As $A$ is a set of independent vertices, we can assume that $w=b_{j} \in B$ and check the following two cases: If $v=a_{h} \in A$, then by definition $h \leq j$, and by construction,

$$
\alpha\left(a_{h}\right)+\alpha\left(b_{j}\right)=i_{h}+k-i_{j} \geq k .
$$

If $v=b_{l} \in B$, then

$$
\alpha\left(b_{l}\right)+\alpha\left(b_{j}\right)=k-i_{l}+k-i_{j} \geq k .
$$

So $\alpha$ is a $k$-cover. The fact that $\left\{a_{j}, b_{j}\right\} \in E$ for each $1 \leq j \leq r$ guarantees that $\alpha$ is a basic $k$-cover. So we may conclude that $\operatorname{dim}(\bar{A}(G)) \geq v_{o}(G)+1$.

Assume now that $\operatorname{dim}(\bar{A}(G))=s+1$. To prove that $\operatorname{dim}(\bar{A}(G)) \leq v_{o}(G)+1$, consider $k>0$ and a basic $k$-cover $\alpha$ as in Lemma 2.7, (b). Denote

$$
\left\{i_{1}, \ldots, i_{r}\right\}=\{\alpha(v): v \in V \text { and } \alpha(v) \leq k / 2\} .
$$

By Lemma 2.7, (b), we have $r \geq s$. We can also assume that $i_{1}>i_{2}>\cdots>i_{r}$. For each $1 \leq j \leq r$, choose a vertex $a_{j} \in V$ such that $\alpha\left(a_{j}\right)=i_{j}$ and denote

$$
A=\left\{a_{1}, \ldots, a_{r}\right\} .
$$


As $\alpha$ is a basic $k$-cover, for each $1 \leq j \leq r$, there exists a vertex $b_{j} \in V$ such that $\alpha\left(a_{j}\right)+\alpha\left(b_{j}\right)=k$. Choose one such $b_{j}$ for each $j$ and denote

$$
B=\left\{b_{1}, \ldots, b_{r}\right\} \text {. }
$$

It is not difficult to see that $A$ is a free parameter set with the partner set $B$, so

$$
v_{o}(G) \geq r \geq s=\operatorname{dim}(\bar{A}(G))-1 .
$$

We recall that the analytic spread of a homogeneous ideal $I \subseteq S$, denoted by $\ell(I)$, is the dimension of its ordinary fiber cone. When $K$ is an infinite field, Northcott and Rees proved in [22] that $\ell(I)$ is the cardinality of a set of minimal generators of a minimal reduction of $I S_{\mathfrak{m}}$, i.e., an ideal $\mathfrak{a} \subseteq S_{\mathfrak{m}}$ is minimal by inclusion and such that there exists $k$ for which $\mathfrak{a}\left(I S_{\mathfrak{m}}\right)^{k}=\left(I S_{\mathfrak{m}}\right)^{k+1}$.

Corollary 2.9 Let $G$ be a bipartite graph. Then

$$
\ell(J(G))=v_{o}(G)+1 .
$$

Proof As said in the preliminaries, in [13, Theorem 5.1.b] the authors showed that $G$ is bipartite if and only if $A(G)$ is a standard graded $S$-algebra. This is equivalent to $A(G)$ being the ordinary Rees algebra of $J(G)$. Therefore, when $G$ is bipartite, $\bar{A}(G)$ is the ordinary fiber cone of $J(G)$, so the corollary follows by Theorem 2.8 .

Before we state the next proposition, let us establish some notation that we will use in its proof. Let $G$ be a bipartite graph with bipartition of the vertex set $V_{1} \cup V_{2}$. In order to compute the ordered matching number, we only need to look at free parameter sets $A_{0} \subseteq V_{1}$ with partner sets $B_{0} \subseteq V_{2}$. Notice that the graph induced by the set of vertices $A_{0} \cup B_{0}$ may not be connected. Denote this graph by $G_{0}$, and denote its connected components by $C_{1}, C_{2}$, and so on. Notice that if $G$ is a tree, then for any vertex $v \notin A_{0} \cup B_{0}$, if there exists an edge $\left\{v, w_{0}\right\}$, with $w_{0}$ in some $C_{i}$, then $\{v, w\}$ is not an edge for any $w \in C_{i}, w \neq w_{0}$. In other words, a vertex outside $G_{0}$ is "tied" to a connected component of $G_{0}$ by at most one edge.

Proposition 2.10 If $G$ is a tree, then $\operatorname{dim} \bar{A}(G)=v_{o}(G)+1=v(G)+1$, where $v(G)$ is the matching number of $G$.

Proof By Remark 2.4 and Theorem 2.8 we only have to prove that $\nu_{o}(G) \geq v(G)$ whenever $G$ is a tree. Choose a maximal free parameter set $A_{0}=\left\{a_{1}, \ldots, a_{r}\right\}$ with partner set $B_{0}=\left\{b_{1}, \ldots, b_{r}\right\}$ and suppose that the matching $M=\left\{\left\{a_{i}, b_{i}\right\}\right\}_{i=1, \ldots, r}$ is not maximal. By a classical result of Berge (for instance, see the book of Lovász and Plummer [18, Theorem 1.2.1]) we get that there must exist an augmenting path in $G$ relative to $M$. As $G$ is bipartite, it is easy to see that this path must be of the form $P=a^{\prime}, b_{i_{1}}, a_{i_{1}}, \ldots, b_{i_{k}}, a_{i_{k}}, b^{\prime}$, and as $A_{0}$ is a free parameter set, the indices must be ordered in the following way: $1 \leq i_{1}<\cdots<i_{k} \leq r$. We will construct a new ordered matching with $r+1$ elements. Notice that $a^{\prime}$ and $b^{\prime}$ are not vertices of $G_{0}$. Denote by $C$ the connected component of $G_{0}$ to which the vertices in $P \cap\left(A_{0} \cup B_{0}\right)$ belong. 
We reorder the connected components so that the $C_{i}$ 's to which $b^{\prime}$ is connected come first, $C$ comes next, and the connected components to which $a^{\prime}$ is connected come last. Inside $C$ we relabel the vertices such that $a_{i_{k}}, a_{i_{k-1}}, \ldots, a_{i_{1}}, a^{\prime}$ are the first $k+1$ vertices with partners $b^{\prime}, b_{i_{k}}, \ldots, b_{i_{2}}, b_{i_{1}}$. It is easy to see now that, as there are no cycles in $G$, we obtain a new ordered matching of cardinality $r+1$, a contradiction.

Given an ideal $I$ of some ring $R$, we recall that the arithmetical rank of $I$ is the integer

$$
\operatorname{ara}(I)=\min \left\{r: \exists f_{1}, \ldots, f_{r} \in R \text { for which } \sqrt{I}=\left(f_{1}, \ldots, f_{r}\right)\right\} .
$$

If $R$ is a factorial domain, geometrically $\operatorname{ara}(I)$ is the minimal number of hypersurfaces that define set-theoretically the scheme $\mathscr{V}(I)$ in $\operatorname{Spec}(R)$. As we said in the beginning of this section, we can obtain interesting upper bounds for this number in the case of monomial ideals of pure codimension 2 in $S_{\mathfrak{m}}$.

Corollary 2.11 Let $K$ be an infinite field, and $G$ a graph. Then

$$
\operatorname{ara}\left(J(G) S_{\mathfrak{m}}\right) \leq v_{o}(G)+1 .
$$

In particular, $\operatorname{ara}\left(J(G) S_{\mathfrak{m}}\right) \leq v(G)+1$.

Proof Let us consider the second Veronese subring of $\bar{A}(G)$, i.e.,

$$
\bar{A}(G)^{(2)}=\bigoplus_{i \geq 0} \bar{A}(G)_{2 i} .
$$

By [13, Theorem 5.1.a] we have $J(G)^{(2 i)}=\left(J(G)^{(2)}\right)^{i}$, so that $\bar{A}(G)^{(2)}$ is the ordinary fiber cone of $J(G)^{(2)}$. Since $\bar{A}(G)$ is finite as an $\bar{A}(G)^{(2)}$-module, the Krull dimensions of $\bar{A}(G)$ and of $\bar{A}(G)^{(2)}$ are the same. Therefore, using Theorem 2.8, we get

$$
v_{o}(G)+1=\operatorname{dim} \bar{A}(G)^{(2)}=\ell\left(J(G)^{(2)}\right)=\ell\left(\left(J(G) S_{\mathfrak{m}}\right)^{(2)}\right) .
$$

By a result in [22, p.151], since $K$ is infinite, the analytic spread of $\left(J(G) S_{\mathfrak{m}}\right)^{(2)}$ is the cardinality of a set of minimal generators of a minimal reduction of it. The radical of such a reduction is clearly the radical of $\left(J(G) S_{\mathfrak{m}}\right)^{(2)}$, i.e., $J(G) S_{\mathfrak{m}}$. So we get the desired inequality.

Remark 2.12 The author of [20] proved that the arithmetical rank of a monomial ideal of pure codimension 2, once localized at $\mathfrak{m}$, is at most $\lfloor n / 2\rfloor+1$, where $n$ is the numbers of variables. But every square-free monomial ideal of codimension 2 is obviously of the form $J(G)$ for some graph on $[n]$. So, since $v(G)$ is at most $\lfloor n / 2\rfloor$, Corollary 2.11 refines the result of [19].

For the next result, let us recall that a set $E^{\prime} \subseteq E(G)=E$ of edges of a graph $G$ is said to be pairwise disconnected if it is a matching and for any two different edges of $E^{\prime}$, there is no edge in $E$ connecting them. 
Corollary 2.13 Let $G$ be a graph for which $v_{o}(G)$ is equal to the maximum size of a set of pairwise disconnected edges. If $K$ is an infinite field, then

$$
\operatorname{ara}\left(J(G) S_{\mathfrak{m}}\right)=v_{o}(G)+1 .
$$

Proof By a result of Katzman [16, Proposition 2.5] the maximum size of a set of pairwise disconnected edges of $G$ provides a lower bound for the Castelnuovo-Mumford regularity of $S / I(G)$. Therefore, $\operatorname{reg}(S / I(G)) \geq v_{o}(G)$. But $J(G)$ is the Alexander dual of $I(G)$, so a result of Terai [24] implies that $\operatorname{pd}(S / J(G)) \geq v_{o}(G)+1$. Now, Lyubeznik showed in [19] that $\operatorname{pd}(S / I)=\operatorname{cd}(S, I)=\operatorname{cd}\left(S_{\mathfrak{m}}, I S_{\mathfrak{m}}\right)$ (cohomological dimension) for any square-free monomial ideal $I$. Since the cohomological dimension provides a lower bound for the arithmetical rank, we get $\operatorname{ara}\left(J(G) S_{\mathfrak{m}}\right) \geq$ $v_{o}(G)+1$. Now we get the conclusion by Corollary 2.11 .

Corollary 2.14 Let $I \subseteq S=K\left[x_{1}, \ldots, x_{n}\right]$ be a square-free monomial ideal of pure codimension 2, and let $d$ be the minimum degree of a non-zero monomial in I. Assume that the field $K$ is infinite. Then

$$
\operatorname{ara}\left(I S_{\mathfrak{m}}\right) \leq \min \{d+1, n-d+1\}
$$

Proof The inequality ara $\left(I S_{\mathfrak{m}}\right) \leq n-d+1$ is well known. One way to see this is by defining the following partial order on the set of the square-free monomials of $S$ :

$$
m \leq n \quad \Longleftrightarrow \quad n \mid m \quad \text { for any square-free monomials } m, n \text { of } S \text {. }
$$

It is easy to see that $S$ is a (nonhomogeneous) algebra with straightening laws (see Bruns and Vetter [6] for the definition) on this poset over $K$. Notice that $I$ comes from a poset ideal. This means that $I=\Omega S$, where $\Omega$ is a subset of the square-free monomials such that

$$
n \in \Omega, \quad m \leq n \quad \Longrightarrow \quad m \in \Omega \text {. }
$$

Then by [6, Proposition 5.20] we get $\operatorname{ara}(I) \leq n-d+1$. This obviously implies that $\operatorname{ara}\left(I S_{\mathfrak{m}}\right) \leq n-d+1$.

To prove the inequality ara $\left(I S_{\mathfrak{m}}\right) \leq d+1$, notice that $I=J(G)$ for a graph $G$ on $[n]\left(\{i, j\}\right.$ is an edge of $G$ if and only if $\left(x_{i}, x_{j}\right)$ is a minimal prime of $\left.I\right)$. Then Corollary 2.11 implies that $\operatorname{ara}\left(I S_{\mathfrak{m}}\right) \leq v(G)+1$. It is well known and easy to show that the matching number is at most the least cardinality of a vertex cover of $G$. It turns out that this number is equal to $d$.

\section{Koszul property and ASL structure of $\bar{A}(G)$}

During an informal conversation at Oberwolfach in 2009, Herzog asked whether $\bar{A}(G)$ is Koszul, provided that $G$ is bipartite. In this section we answer this question positively, showing even more: if $G$ is bipartite, then $\bar{A}(G)$ has a structure of homogeneous ASL. 
Algebras with straightening laws (ASLs for short) were introduced by De Concini, Eisenbud, and Procesi in [7]. These algebras provide an unified treatment of both algebraic and geometric objects that have a combinatorial nature. For example, the coordinate rings of some classical algebraic varieties (such as determinantal rings and Pfaffian rings) have an ASL structure. For more details on this topic, the reader can consult [6]. First, we will recall the definition of homogeneous ASL on posets.

Let $(P,<)$ be a finite poset and denote by $K[P]=K\left[X_{p}: p \in P\right]$ the polynomial ring over $K$ whose variables correspond to the elements of $P$. Denote by $I_{P}$ the following monomial ideal of $K[P]$ :

$$
I_{P}=\left(X_{p} X_{q}: p \text { and } q \text { are incomparable elements of } P\right)
$$

Definition 3.1 Let $A=K[P] / I$, where $I$ is a homogeneous ideal with respect to the standard grading. The graded algebra $A$ is called a homogeneous ASL on $P$ if

(ASL1) The residue classes of the monomials not in $I_{P}$ are linearly independent in $A$.

(ASL2) For every $p, q \in P$ such that $p$ and $q$ are incomparable, the ideal $I$ contains a polynomial of the form

$$
X_{p} X_{q}-\sum \lambda X_{s} X_{t}
$$

with $\lambda \in K, s, t \in P, s \leq t, s<p$, and $s<q$. The above sum is allowed to run on the empty set.

The polynomials in (ASL2) give a way of rewriting in $A$ the product of two incomparable elements. These relations are called the straightening relations or straightening laws.

A total order $<^{\prime}$ on $P$ is called a linear extension of the poset $(P,<)$ if $x<y$ implies $x<^{\prime} y$. It is known that if $\tau$ is a revlex term order with respect to a linear extension of $<$, then the polynomials in (ASL2) form a Gröbner basis of $I$, and $\operatorname{in}_{\tau}(I)=I_{P}$.

We will prove now that when $G$ is a bipartite graph, $\bar{A}(G)$ has an ASL structure. Let us first fix some notation. Let $G$ be a bipartite graph with the partition of the vertex set $[n]=A \cup B$ and suppose that $|A| \leq|B|$. We denote by $\mathscr{C}(G)$ the set of 1 -covers of $G$ which take values in $\{0,1\}$ (not necessarily basic). Equivalently, $\mathscr{C}(G)$ is the set of vertex covers of $G$. We define on $\mathscr{C}(G)$ the following partial order: Given $\alpha, \beta \in \mathscr{C}(G)$, we say that

$$
\alpha \leq \beta \quad \Longleftrightarrow \quad \alpha(a) \leq \beta(a) \quad \forall a \in A \quad \text { and } \quad \alpha(b) \geq \beta(b) \quad \forall b \in B
$$

Actually, with this partial order, $\mathscr{C}(G)$ becomes a distributive lattice, as we are going to explain. We recall that a poset $\mathscr{L}$ is a lattice if every two elements $l, l^{\prime} \in L$ have a supremum, denoted by $l \vee l^{\prime}$, and an infimum, denoted by $l \wedge l^{\prime}$. Furthermore we say that $\mathscr{L}$ is distributive if $l \vee\left(l^{\prime} \wedge l^{\prime \prime}\right)=\left(l \vee l^{\prime}\right) \wedge\left(l \vee l^{\prime \prime}\right)$. 
Remark 3.2 The poset structure we gave to $\mathscr{C}(G)$ actually confers a distributive lattice structure to $\mathscr{C}(G)$. Given $\alpha, \beta \in \mathscr{C}(G)$, set

$$
\begin{aligned}
& (\alpha \vee \beta)(v)= \begin{cases}\max \{\alpha(v), \beta(v)\} & \text { if } v \in A, \\
\min \{\alpha(v), \beta(v)\} & \text { if } v \in B ;\end{cases} \\
& (\alpha \wedge \beta)(v)= \begin{cases}\min \{\alpha(v), \beta(v)\} & \text { if } v \in A, \\
\max \{\alpha(v), \beta(v)\} & \text { if } v \in B .\end{cases}
\end{aligned}
$$

Clearly, $\alpha \vee \beta$ and $\alpha \wedge \beta$ belong to $\mathscr{C}(G)$ and are respectively the supremum and the infimum of $\alpha$ and $\beta$. Moreover it is straightforward to verify the distributivity of these operations.

Let $\mathscr{P}(G)$ be the set of basic 1-covers of $G$. One has $\mathscr{P}(G) \subseteq \mathscr{C}(G)$, so the partial order on $\mathscr{C}(G)$ induces a poset structure also on $\mathscr{P}(G)$. Unfortunately, even if $\alpha$ and $\beta$ are basic, it may happen that $\alpha \vee \beta$ or $\alpha \wedge \beta$ are not basic. So, in general, $\mathscr{P}(G)$ does not inherit the lattice structure from $\mathscr{C}(G)$.

Remark 3.3 Notice that the poset structure on $\mathscr{P}(G)$ can be read off only from $A$ or $B$. In fact, if $\alpha$ and $\beta$ are basic 1-covers, we have $\alpha(a) \leq \beta(a) \forall a \in A \Longleftrightarrow$ $\alpha(b) \geq \beta(b) \forall b \in B$. Therefore, for all $\alpha, \beta \in \mathscr{P}(G)$, we have

$$
\alpha \leq \beta \quad \Longleftrightarrow \quad \alpha(a) \leq \beta(a) \quad \forall a \in A \quad \Longleftrightarrow \quad \alpha(b) \geq \beta(b) \quad \forall b \in B .
$$

For any $\alpha, \beta \in \mathscr{C}(G)$, it is easy to check the following equality:

$$
\alpha+\beta=\alpha \wedge \beta+\alpha \vee \beta
$$

where the sum is component-wise. The above equality translates to a relation among the generators of $\bar{A}(G)$ in the following way. Denote $R$ the polynomial ring $K[\mathscr{P}(G)]=K\left[X_{\alpha}: \alpha \in \mathscr{P}(G)\right]$. We have the following natural presentation of the $K$-algebra $\bar{A}(G)$ :

$$
\begin{aligned}
\Phi: \quad R & \longrightarrow \bar{A}(G), \\
X_{\alpha} & \longmapsto x_{1}^{\alpha(1)} \cdots x_{n}^{\alpha(n)} t .
\end{aligned}
$$

For simplicity, we set $X_{\alpha \vee \beta}$ (respectively $X_{\alpha \wedge \beta}$ ) to be 0 (as elements of $R$ ) whenever they are not basic 1-covers. Using the above convention, it is obvious that the polynomial

$$
X_{\alpha} X_{\beta}-X_{\alpha \wedge \beta} X_{\alpha \vee \beta}
$$

belongs to the kernel of $\Phi$ for any pair of basic 1-covers $\alpha$ and $\beta$. The main result of this section is the following theorem. 
Theorem 3.4 Let $G$ be a bipartite graph. The algebra $\bar{A}(G)$ has a homogeneous ASL structure on $\mathscr{P}(G)$ over $K$. With the above notation, the straightening relations are $\Phi\left(X_{\alpha}\right) \Phi\left(X_{\beta}\right)= \begin{cases}\Phi\left(X_{\alpha \wedge \beta}\right) \Phi\left(X_{\alpha \vee \beta}\right) & \text { if both } \alpha \vee \beta \text { and } \alpha \wedge \beta \text { are basic 1-covers } \\ 0 & \text { otherwise, }\end{cases}$ for any incomparable basic 1-covers $\alpha$ and $\beta$. In particular, we have $\operatorname{ker} \Phi=\left(X_{\alpha} X_{\beta}-X_{\alpha \wedge \beta} X_{\alpha \vee \beta}: \alpha\right.$ and $\beta$ are incomparable basic 1-covers $)$.

Before proving Theorem 3.4, we will prove the following lemma.

Lemma 3.5 Let $G$ be a bipartite graph. Set

$$
\mathscr{M}=\left\{U \in R: U=X_{\alpha_{1}} \cdots X_{\alpha_{d}}, d \in \mathbb{N}, \alpha_{1} \leq \cdots \leq \alpha_{d}\right\} .
$$

The subset $\Phi(\mathscr{M}) \subseteq \bar{A}(G)$ consists of linearly independent elements of $\bar{A}(G)$.

Proof First of all, we will show that $\Phi(U) \neq 0$ for any $U \in \mathscr{M}$. By contradiction, suppose that there are basic 1-covers $\alpha_{1} \leq \cdots \leq \alpha_{d}$ such that $U=X_{\alpha_{1}} \cdots X_{\alpha_{d}}$ is in the kernel of $\Phi$. In other words, the $d$-cover $\gamma$ that associates to a vertex $v$ the value $\gamma(v)=\alpha_{1}(v)+\cdots+\alpha_{d}(v)$ is nonbasic. So there exists a vertex $v_{0}$ of $G$ such that $\gamma\left(v_{0}\right)+\gamma(w)>d$ for any $w$ adjacent to $v_{0}$. Let us assume that $v_{0} \in A$ (otherwise the issue is similar). Set $q=\min \left\{i=1, \ldots, d: \alpha_{i}\left(v_{0}\right)=1\right\}$; if $\alpha_{i}\left(v_{0}\right)=0$ for any $i$, we set $q=d+1$. Since $\alpha_{q}$ is a basic 1-cover, there exists a vertex $w_{0}$ adjacent to $v_{0}$ such that $\alpha_{q}\left(v_{0}\right)+\alpha_{q}\left(w_{0}\right)=1$. As $\alpha_{1} \leq \cdots \leq \alpha_{d}$, we have $\alpha_{i}\left(v_{0}\right)=0$ for all $i<q$ and $\alpha_{j}\left(w_{0}\right)=0$ for all $j \geq q$ (because $w_{0} \in B$ and $\alpha_{q}\left(w_{0}\right)=0$ ). This implies that

$$
\gamma\left(v_{0}\right)+\gamma\left(w_{0}\right)=\sum_{i=q}^{d} \alpha_{i}\left(v_{0}\right)+\sum_{j=1}^{q-1} \alpha_{j}\left(w_{0}\right)=(d-q+1)+(q-1)=d,
$$

a contradiction.

Since $\left\{x_{1}^{\gamma(1)} \cdots x_{n}^{\gamma(n)}: \gamma\right.$ is a basic $d$-cover, $\left.d \in \mathbb{N}\right\}$ is a $K$-basis of $\bar{A}(G)$, it is enough to show that $\Phi(U) \neq \Phi(V)$ whenever $U$ and $V$ are different elements of $\mathscr{M}$. Suppose that $U=X_{\alpha_{1}} \cdots X_{\alpha_{d}}$ and $V=X_{\beta_{1}} \cdots X_{\beta_{e}}$. If $d \neq e$, using the facts proved above, we have $\Phi(U) \neq \Phi(V)$. Thus consider the case $d=e$. Since $U \neq V$, there exists an index $j=1, \ldots, d$ such that $\alpha_{j} \neq \beta_{j}$. So there exists a vertex $v_{0}$ of $G$ such that $\alpha_{j}\left(v_{0}\right) \neq \beta_{j}\left(v_{0}\right)$. Let us assume that $v_{0} \in A, \alpha_{j}\left(v_{0}\right)=0$ and $\beta_{j}\left(v_{0}\right)=1$. The other cases are similar. Furthermore, up to a relabeling we can assume that $v_{0}=1$. Since $\alpha_{1} \leq \cdots \leq \alpha_{d}$ and $\beta_{1} \leq \cdots \leq \beta_{d}$, we get $\alpha_{i}(1)=0$ for all $i \leq j$ and $\beta_{h}(1)=1$ for all $h \geq j$. So we have that $\Phi(U)$ has degree less than or equal to $d-j$ with respect to $x_{1}$, whereas $\Phi(V)$ has degree at least $d-j+1$ with respect to it. Therefore they cannot be equal.

Proof of Theorem 3.4 We have seen that $\bar{A}(G)=R / \operatorname{ker} \Phi$. Because $G$ is bipartite, the graded $K$-algebra $\bar{A}(G)$ is generated by the elements $x^{\alpha}=x_{1}^{\alpha(1)} \cdots x_{n}^{\alpha(n)}$, with $\alpha$ a basic 1 -cover. Moreover the degree of $x^{\alpha}$ is 1 if $\alpha$ is a basic 1 -cover. So $\operatorname{ker} \Phi$ 
is homogeneous with respect to the standard grading of $R$. We need to see now that (ASL1) and (ASL2) are satisfied.

The first condition follows by Lemma 3.5. From the discussion preceding Theorem 3.4 we get that the polynomials $X_{\alpha} X_{\beta}-X_{\alpha \wedge \beta} X_{\alpha \vee \beta}$ belong to ker $\Phi$. By construction $\alpha \wedge \beta<\alpha \vee \beta, \alpha \wedge \beta<\alpha$, and $\alpha \wedge \beta<\beta$ (whenever $\alpha \wedge \beta$ and $\alpha \vee \beta$ are basic 1-covers). So (ASL2) holds as well. The last part of the statement follows immediately from [6, Proposition 4.2].

As we said in the beginning of this section, the homogeneous ASL structure of $\bar{A}(G)$ implies that the straightening relations form a quadratic Gröbner basis. This implies the following corollary.

Corollary 3.6 If $G$ is a bipartite graph, then $\bar{A}(G)$ is a Koszul algebra.

Remark 3.7 Independently and by different methods, Rinaldo showed in [23, Corollary 3.9] a particular case of Corollary 3.6. Namely he proved that $\bar{A}(G)$ is Koszul, provided that $G$ is a bipartite graph satisfying the weak square condition (see the definition below). Actually we will show in Corollary 3.8 that for such a graph, $\bar{A}(G)$ is even a Hibi ring.

A special class of algebras with straightening laws are the so-called Hibi rings. They were constructed in [14] as an example of integral ASLs. The poset that supports their structure is a distributive lattice $\mathscr{L}$, and the straightening relations are given for any two incomparable elements $p, q \in \mathscr{L}$ by

$$
X_{p} X_{q}-X_{p \wedge q} X_{p \vee q}
$$

In [1] the following property for bipartite graphs was introduced, which was then extended in [2] for any graph. A graph $G$ is said to have the weak square condition (WSC for short) if for every vertex $v \in V$, there exists an edge $\{v, w\} \in E$ containing it such that

$$
\left.\begin{array}{r}
\left\{v, v^{\prime}\right\} \in E \\
\left\{w, w^{\prime}\right\} \in E
\end{array}\right\} \quad \Rightarrow \quad\left\{v^{\prime}, w^{\prime}\right\} \in E .
$$

We have the following corollary.

Corollary 3.8 Let $G$ be a bipartite graph. The following are equivalent:

(i) $G$ satisfies the WSC;

(ii) $\bar{A}(G)$ is a domain;

(iii) $\bar{A}(G)$ is a Hibi ring on $\mathscr{P}(G)$ over $K$.

Proof The equivalence between (i) and (ii) was already proved in [1, Theorem 1.9], and we present it here only for completeness. The fact that (iii) implies (ii) was proved by Hibi in the same paper where he introduced these algebras (see [14, p. 100]). So we only need to prove that (ii) implies (iii).

For every $\alpha, \beta \in \mathscr{P}(G)$ that are incomparable, we must have $X_{\alpha} X_{\beta}-X_{\alpha \wedge \beta} X_{\alpha \vee \beta} \in$ $\operatorname{ker} \Phi$ by Theorem 3.4. Since $\bar{A}(G)$ is a domain, then both $\alpha \wedge \beta$ and $\alpha \vee \beta$ have to 
be basic 1-covers. In other words, in this case the poset $\mathscr{P}(G)$ inherits the latticestructure from $\mathscr{C}(G)$. So by [14, p. 100] and by Theorem 3.4 we conclude.

A classical structure theorem of Birkhoff [3, p. 59] states that for each distributive lattice $\mathscr{L}$, there exists a unique poset $P$ such that $\mathscr{L}=J(P)$, where $J(P)$ is the set of poset ideals of $P$, ordered by inclusion. By Corollary 3.8 we have that if a bipartite graph $G$ satisfies the WSC, then the poset of basic 1-covers $\mathscr{P}(G)$ is a distributive lattice. So by Birkhoff's result there exists a unique poset $P_{G}$ such that $\mathscr{P}(G)=J\left(P_{G}\right)$. We use now another result of Hibi which describes completely the Gorenstein Hibi rings (see [14, p. 105]) to obtain the following corollary.

Corollary 3.9 Let $G$ be a bipartite graph satisfying the WSC. The following conditions are equivalent:

(i) $\bar{A}(G)$ is Gorenstein;

(ii) the poset $P_{G}$ defined above is pure.

We want to close this section showing some tools to deduce properties of $\bar{A}(G)$ from the combinatorics of $\mathscr{P}(G)$. In particular, we will focus on the CohenMacaulayness of $\bar{A}(G)$, but one can $\operatorname{read}$ off $\mathscr{P}(G)$ also the dimension, the multiplicity, and the Hilbert series of $\bar{A}(G)$.

The main technique is to consider the "canonical" initial ideal of the ideal defining $\bar{A}(G)$. Let $I$ be the ideal, which we described above in terms of its generators, such that $\bar{A}(G)=R / I$ (recall that $R=K[\mathscr{P}(G)]$ ). Denote by in $(I)$ the initial ideal of it with respect to a degrevlex term order associated to a linear extension of the partial order on $\mathscr{P}(G)$. From the results of this section it follows that $\operatorname{in}(I)$ is a squarefree monomial ideal, so we can associate to it a simplicial complex $\Delta=\Delta(\operatorname{in}(I))$. Moreover it is easy to show that $\Delta$ is the order complex of $\mathscr{P}(G)$, i.e., its faces are the chains of $\mathscr{P}(G)$.

Example 3.10 Non-Cohen-Macaulay $\bar{A}(G)$. Let $G$ be a path of length $n-1 \geq 5$. So $G$ is a graph on $n$ vertices with edges

$$
\{1,2\},\{2,3\}, \ldots,\{n-1, n\} .
$$

For any $i=1, \ldots,\lfloor n / 2\rfloor$, define the basic 1 -cover

$$
\alpha_{i}(j)= \begin{cases}1 & \text { if } j=2 k \text { and } k \leq i \\ 1 & \text { if } j=2 k-1 \text { and } k>i \\ 0 & \text { otherwise }\end{cases}
$$

Then define also the basic 1-cover

$$
\beta(j)= \begin{cases}1 & \text { if } j=1,3 \text { or } j=2 k \text { with } k \geq 2 \\ 0 & \text { otherwise }\end{cases}
$$


It is easy to verify that $\alpha_{1} \leq \alpha_{2} \leq \alpha_{3} \leq \cdots \leq \alpha_{\lfloor n / 2\rfloor}$ and $\beta \leq \alpha_{3} \leq \cdots \leq \alpha_{\lfloor n / 2\rfloor}$ are maximal chains of $\mathscr{P}(G)$. So $\mathscr{P}(G)$ is not pure. Therefore the order complex of $\mathscr{P}(G)$ is not pure. So $\bar{A}(G)$ is not an equidimensional ring by [15, Corollary 1]. In particular, if $G$ is a path of length at least $5, \bar{A}(G)$ is not Cohen-Macaulay.

Before stating the following result, we recall some notion regarding posets. A poset $P$ is bounded if it has a least and a greatest element. An element $x \in P$ covers $y \in P$ if $y \leq x$ and there does not exist $z \in P$ with $y<z<x$. The poset $P$ is said to be locally upper semi-modular if whenever $v_{1}$ and $v_{2}$ cover $u$ and $v_{1}, v_{2}<v$ for some $v$ in $P$, then there exists $t \in P, t \leq v$, which covers $v_{1}$ and $v_{2}$.

Theorem 3.11 Let $G$ be a bipartite graph, and $A \cup B$ a bipartition of the vertex set with $|A| \leq|B|$. Let $\Delta$ be the order complex of $\mathscr{P}(G)$. If $\operatorname{rank}(\mathscr{P}(G))=|A|$, then the following are equivalent:

(i) $\bar{A}(G)$ is equidimensional;

(ii) $\mathscr{P}(G)$ is a pure poset;

(iii) $\Delta$ is shellable;

(iv) $\bar{A}(G)$ is Cohen-Macaulay.

Proof (iv) $\Rightarrow$ (i) is well known. As the Cohen-Macaulayness of $R / \operatorname{in}(I)$ implies the Cohen-Macaulayness of $R / I \cong \bar{A}(G)$, (iii) $\Rightarrow$ (iv) is also true. (i) $\Rightarrow$ (ii) follows by [15, Corollary 1].

(ii) $\Rightarrow$ (iii) To prove that $\Delta$ is shellable, we will use a result of Björner (see [4, Theorem 6.1]), stating that it is enough to show that $\mathscr{P}(G)$ is a bounded locally upper semimodular poset. The poset $\mathscr{P}(G)$ is obviously bounded, so let $\alpha$ and $\beta$ be two elements of $\mathscr{P}(G)$ which cover $\gamma$. The fact that $\operatorname{rank}(\mathscr{P}(G))=|A|$, together with the pureness of $\mathscr{P}(G)$, implies that for a basic 1-cover $\xi$, we have $\operatorname{rank}(\xi)=\sum_{v \in A} \xi(v)$. If $\alpha$ and $\beta$ cover $\gamma$, since all the unrefinable chains between two comparable elements of a bounded pure complex have the same length, it follows that $s=\operatorname{rank}(\alpha)=\operatorname{rank}(\beta)=\operatorname{rank}(\gamma)+1$. But $\gamma(v) \leq \min \{\alpha(v), \beta(v)\}$ for each $v \in A$, so if we look at the rank of the elements involved, we obtain $\gamma(v)=\min \{\alpha(v), \beta(v)\}$ for all $v \in A$. Consider the (not necessarily basic) 1-cover, defined at the beginning of this section: $\alpha \vee \beta$. It is easy to see that, to make it basic, we can reduce its value at some vertex in $B$, and not in $A$. Let $\delta$ be the basic 1-cover obtained from $\alpha \vee \beta$. Then

$$
\operatorname{rank}(\delta)=\sum_{v \in A} \delta(v)=\sum_{v \in A}(\alpha \vee \beta)(v)=s+1,
$$

which implies that $\delta$ covers $\alpha$ and $\beta$.

By Theorems 2.8 and 3.4, we have that $\operatorname{rank}(\mathscr{P}(G))=v_{o}(G)$, so the hypothesis of the theorem concerns just the combinatorics of the graph.

We showed in [1] that if $\bar{A}(G)$ is a domain, then $\bar{A}(G)$ is Cohen-Macaulay. Given the above example and theorem, it is natural to ask the following questions: Can $\bar{A}(G)$ be Cohen-Macaulay and not a domain? Are there examples of graphs for which 
$\mathscr{P}(G)$ is pure but $\bar{A}(G)$ is not Cohen-Macaulay? Both answers are positive, and they are provided by the following examples.

\section{Example 3.12}

1. $\bar{A}(G)$ Cohen-Macaulay but not domain. Consider the graph $G$ on seven vertices below:
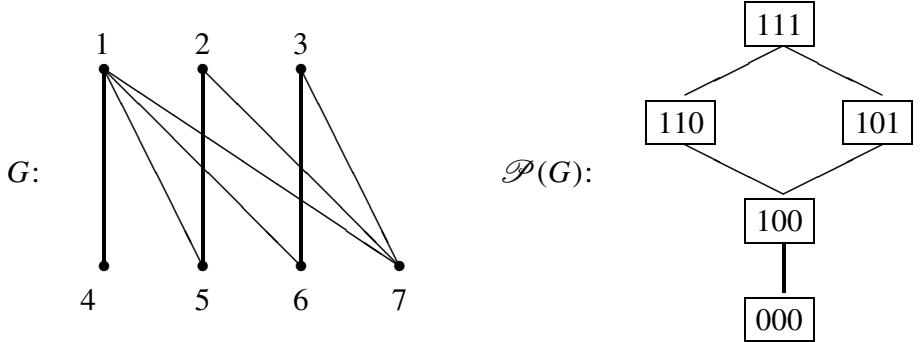

It is easy to see that $G$ does not satisfy the WSC. We order the basic 1-covers component-wise with respect to the values they take on the vertex set $\{1,2,3\}$. It is clear from the Hasse diagram above that $\mathscr{P}(G)$ is pure. Moreover $\operatorname{rank}(\mathscr{P}(G))=$ $v_{o}(G)=3=|A|$, so Theorem 3.11 implies that $\bar{A}(G)$ is Cohen-Macaulay.

2. $\mathscr{P}(G)$ pure but $\bar{A}(G)$ not Cohen-Macaulay. Consider the graph $G$ in the picture below. It is not difficult to see that it has only six basic 1-covers. On the right you can see the Hasse diagram of the poset $\mathscr{P}(G)$. The values written next to the vertices represent the basic 1-cover written in bold on the right. Notice that the partial order is defined component-wise with respect to the values taken on the "upper" vertices of $G$.
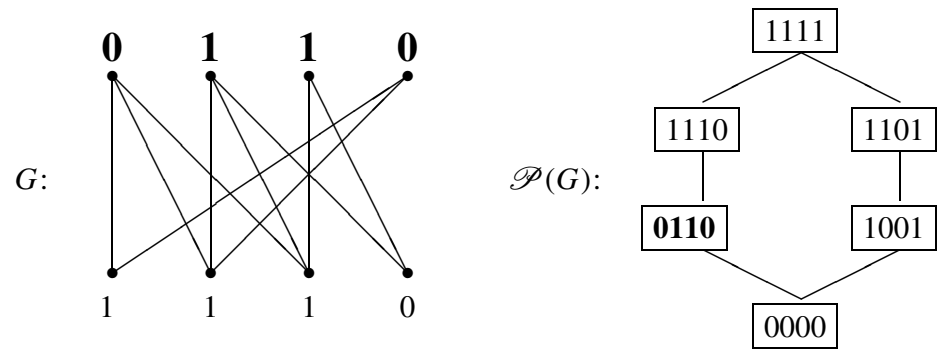

The poset $\mathscr{P}(G)$ is pure, but the ordered complex of it is not strongly connected. Then $I$ has an initial ideal not connected in codimension 1, so [25, Corollary 2.13] implies that $\bar{A}(G)$ is not Cohen-Macaulay.

\section{Cohen-Macaulayness of edge ideals of graphs with the weak square condition}

An interesting open problem, far from being solved, is to characterize in a combinatorial fashion all the Cohen-Macaulay graphs. The authors of [12] gave a complete 
answer when $G$ is bipartite. On the other hand, if $G$ is Cohen-Macaulay, then it is unmixed, and for bipartite unmixed graphs, $\bar{A}(G)$ is the ordinary fiber cone of an ideal generated in one degree, so it is a domain. This means that a bipartite CohenMacaulay graph satisfies the WSC. Since many of these graphs are not bipartite (see [2] for details), a natural extension of the theorem of Herzog and Hibi would be to characterize all the graphs satisfying the WSC which are Cohen-Macaulay. We are able to do this defining for each graph $G$ a "nicer" graph $\pi(G)$. This association behaves like a projection.

We start with a definition that makes sense by [2, Lemma 2.1].

Definition 4.1 We say that an edge $\{i, j\}$ of $G$ is a transversal edge if one of the following equivalent conditions is satisfied:

(i) for any basic 1-cover $\alpha$ of $G$, we have $\alpha(i)+\alpha(j)=1$;

(ii) for any basic $k$-cover $\alpha$ of $G$, we have $\alpha(i)+\alpha(j)=k$;

(iii) if $\left\{i, i^{\prime}\right\}$ and $\left\{j, j^{\prime}\right\}$ are edges of $G$, then $\left\{i^{\prime}, j^{\prime}\right\}$ is an edge of $G$ as well (in particular, $\left.i^{\prime} \neq j^{\prime}\right)$.

Notice that a graph satisfies the WSC if and only if every vertex belongs to a transversal edge. We recall that these graphs are of interest because they are exactly those graphs for which $\bar{A}(G)$ is a domain. In [2] the authors constructed from $G$ a graph $G^{0-1}$, possibly with isolated vertices, in order to characterize the graphs for which all the symbolic powers of $J(G)$ are generated in one degree. We recall the definition:

(1) $V\left(G^{0-1}\right)=V(G)$;

(2) $E\left(G^{0-1}\right)=\{\{i, j\} \in E(G):\{i, j\}$ is a transversal edge of $G\}$.

It was proved in [2] that for any $G$, the graph $G^{0-1}$ is the disjoint union of some complete bipartite graphs $K_{r, s}$ (with $s \geq r \geq 1$ ) and some isolated points. Moreover, $G^{0-1}$ has no isolated vertices if and only if $G$ satisfies the WSC.

We construct a new graph, denoted by $\pi(G)$, as follows: assume that

$$
G^{0-1}=\left(\bigcup_{i=1}^{m} K_{r_{i}, s_{i}}\right) \bigcup\left(\bigcup_{j=1}^{t}\left\{v_{j}\right\}\right),
$$

where the unions are disjoint unions of graphs, $r_{i} \geq s_{i} \geq 1$, and $v_{j} \in V(G)$. Denote by $\left(A_{i}, B_{i}\right)$ the bipartition of $K_{r_{i}, s_{i}}$ and choose, for each $i$, one vertex $a_{i} \in A_{i}$ and one vertex $b_{i} \in B_{i}$. We define the vertex set of $\pi(G)$ as

$$
V(\pi(G))=\left\{a_{i}, b_{i}, v_{j}: i=1, \ldots, m \text { and } j=1, \ldots, t\right\} .
$$

The graph $\pi(G)$ will be the restriction of $G$ to $V(\pi(G)) \subseteq V(G)$. In particular,

$$
E(\pi(G))=\{\{i, j\} \in E(G): i, j \in V(\pi(G))\} .
$$

By [2, Lemma 2.6] the definition of $\pi(G)$ does not depend on the choice of the vertices $a_{i}$ and $b_{i}$. This is because, for any two elements $U$ and $W$ of the set 
$\left\{A_{i}, B_{i},\left\{v_{j}\right\}: i=1, \ldots, m\right.$ and $\left.j=1, \ldots, t\right\}$, the existence of an edge from $U$ to $W$ is equivalent to the fact that the induced subgraph of $G$ on the vertices of $U \cup W$ is bipartite complete. The notation $\pi$ comes from the fact that the operator $\pi$ is a projection, in the sense that $\pi(\pi(G))=\pi(G)$.

In the following picture we present an example of how this construction works:

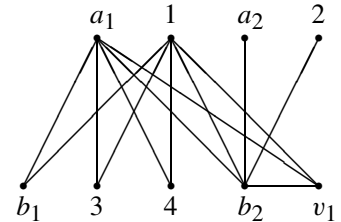

$G$

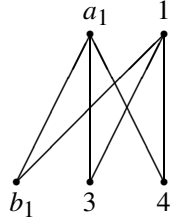

$$
G^{0-1}
$$

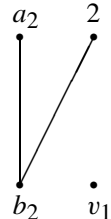

$v$

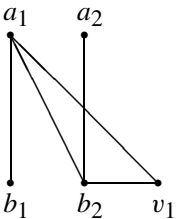

$\pi(G)$

The following result is one of the reasons for introducing $\pi(G)$.

Proposition 4.2 For every graph $G$, there is a well-defined 1-1 correspondence

$$
\pi:\{\text { basic covers of } G\} \longrightarrow\{\text { basic covers of } \pi(G)\}
$$

that associates to a basic $k$-cover $\alpha$ of $G$ the basic $k$-cover $\pi(\alpha)$ of $\pi(G)$, with $\pi(\alpha)(v)=\alpha(v)$ for all $v \in V(\pi(G))$. Moreover, this correspondence induces a graded isomorphism

$$
\bar{A}(G) \cong \bar{A}(\pi(G))
$$

Proof Using the fact that the edges between each $A_{i}$ and $B_{i}$ are transversal, it is straightforward to check that $\alpha$ has the same value on all vertices in $A_{i}$ (respectively in $B_{i}$ ) for every $i=1, \ldots, m$. This implies that the definition of $\pi$ does not depend on the choice of $a_{i}$ and $b_{i}$ for any $i$. It is easy to see that $\pi$ is a bijection between the basic $k$-covers of $G$ and those of $\pi(G)$; moreover, this operation is compatible with the multiplicative structure on $\bar{A}(G)$ and of $\bar{A}(\pi(G))$. Therefore, we also have a graded isomorphism between the algebras $\bar{A}(G)$ and $\bar{A}(\pi(G))$.

\section{Remark 4.3}

(1) The previous proposition provides another proof of the fact that $\bar{A}(G)$ is a Hibi ring when $G$ is a bipartite graph satisfying the WSC. In fact in this case $\pi(G)$ is unmixed bipartite, so it is known that $\bar{A}(\pi(G))$ is a Hibi ring (for instance, see [1, Theorem 3.3]).

(2) Proposition 4.2 shows also that $\mathscr{P}(G)=\mathscr{P}(\pi(G))$. So in order to study $\mathscr{P}(G)$, it can be convenient to pass to the projection and work on a graph with less vertices.

In some cases, $\pi(G)=G$, for instance, if $G$ is a cycle on $n \neq 4$ vertices. The usefulness of $\pi(G)$ arises especially when $G$ satisfies the WSC. As we already said in the above remark, in this case, $\pi(G)$ is unmixed. Less trivially, we can strengthen this fact, but first we need a technical lemma. 
Lemma 4.4 Let $G$ be a graph satisfying the WSC. Then there exists a unique perfect matching $M=\left\{\left\{u_{i}, v_{i}\right\}: i=1, \ldots, r\right\}$ of $\pi(G)$, where $r=|\pi(G)| / 2$. Moreover, it is possible to label the vertices of $\pi(G)$ in such a way that $\left\{v_{1}, \ldots, v_{r}\right\}$ is an independent set of vertices of $\pi(G)$ and that the relation $v_{i} \prec v_{j}$ if and only $\left\{u_{i}, v_{j}\right\}$ is an edge defines a partial order on $V=\left\{v_{1}, \ldots, v_{r}\right\}$.

Proof Since $G$ satisfies the WSC, $G^{0-1}$ has no isolated points, so we obtain a perfect matching $M=\left\{\left\{u_{i}, v_{i}\right\}: i=1, \ldots, r\right\}$ directly by construction. Moreover, since the edges of $M$ are transversal, it immediately follows that for each 1-cover $\alpha$ of $\pi(G)$, we have $\sum_{v \in \pi(G)} \alpha(v)=r$. This implies that if $N$ is another perfect matching of $\pi(G)$, then the $r$ edges of $N$ must be transversal. But the only transversal edges of $\pi(G)$ are those of $M$, and therefore, $M=N$.

We prove now that we can assume that $\left\{v_{1}, \ldots, v_{r}\right\}$ is an independent set of vertices. In fact, suppose that there exist $i<j$ such that $\left\{v_{i}, v_{j}\right\}$ is an edge and take the least $j$ with this property. First notice that there exists no edge $\left\{u_{j}, v_{k}\right\}$ of $\pi(G)$ with $k<j$. The existence of such an edge would imply that also $\left\{v_{k}, v_{i}\right\}$ is an edge (as $\left\{u_{j}, v_{j}\right\}$ is transversal), and this would contradict the minimality of $j$. Now switch $v_{j}$ and $u_{j}$. As we have seen that there are no edges $\left\{u_{j}, v_{k}\right\}$ with $k<j$, we can proceed with the same argument and assume that $\left\{v_{1}, \ldots, v_{r}\right\}$ is an independent set of vertices.

To conclude, we have to show that the relation

$$
v_{i} \prec v_{j} \quad \Longleftrightarrow \quad\left\{u_{i}, v_{j}\right\} \text { is an edge of } \pi(G)
$$

defines a partial order on $V$.

(1) Reflexivity is obvious.

(2) Transitivity is straightforward because $\left\{u_{i}, v_{i}\right\}$ is a transversal edge of $\pi(G) \forall i=$ $1, \ldots, r$.

(3) Anti-symmetry: suppose that there exist $i \neq j$ such that $v_{i} \prec v_{j}$ and $v_{j} \prec v_{i}$. Then $\left\{u_{i}, v_{j}\right\}$ and $\left\{u_{j}, v_{i}\right\}$ are both edges of $\pi(G)$. This contradicts [2, Lemma 2.6, point (3)].

We recall that if $I \subseteq S$ is a square-free monomial ideal, we can associate to it the simplicial complex $\Delta(I)$ on the set $[n]$ such that $\left\{i_{1}, \ldots, i_{s}\right\}$ belongs to $\Delta(I)$ if and only if $x_{i_{1}} \cdots x_{i_{s}}$ does not belong to $I$.

To prove the next result, we need a theorem from [21] that we are going to state in the case of graphs. We recall that a graph $G$ has a perfect matching of König type if it has a perfect matching of cardinality ht $(I(G))$.

Theorem 4.5 (Morey, Reyes, and Villarreal [21, Theorem 2.8]) Let $G$ be an unmixed graph which admits a matching of König type. Assume that for any vertex $v$, the induced subgraph on all the vertices of $G$ but $v$ has a leaf. Then $\Delta(I(G))$ is shellable.

Thus we are ready to show the following. 
Theorem 4.6 Let $G$ be a graph satisfying the WSC, and let $\Delta=\Delta(I(\pi(G))$. Then $\Delta$ is shellable. In particular, $\pi(G)$ is a Cohen-Macaulay graph.

Proof We want to use Theorem 4.5. It is clear that $\pi(G)$ is unmixed because it has a perfect matching of transversal edges. Furthermore, such a matching is obviously of König type. It remains to show that for any $v \in V(\pi(G))$, the induced subgraph of $\pi(G)$ on $V(\pi(G)) \backslash\{v\}$ has a leaf. Label the vertices of $\pi(G)$ as in Lemma 4.4 and in such a way that $v_{i} \prec v_{j}$ implies $i \leq j$. Since $v_{1}$ is a leaf, the only problem could arise when we remove from $\pi(G)$ either $u_{1}$ or $v_{1}$. If we remove $u_{1}$, then $v_{2}$ becomes a leaf, so we must show that the graph induced by $\pi(G)$ on $V(\pi(G)) \backslash\left\{v_{1}\right\}$ has a leaf.

Suppose that there are no leaves. Then, denoting by $r=|V(\pi(G))| / 2$, we can choose the minimum $i$ such that $\left\{u_{i}, u_{r}\right\}$ is an edge (because $u_{r}$ is not a leaf, and by Lemma 4.4 these are the only possible edges, different from $\left\{u_{r}, v_{r}\right\}$, containing $u_{r}$ ). We claim that $i=1$. If not, since $v_{i}$ is not a leaf, there exists $j<i$ such that $\left\{u_{j}, v_{i}\right\}$ is an edge. But, since $\left\{u_{i}, v_{i}\right\}$ is a transversal edge, it follows that $\left\{u_{j}, u_{r}\right\}$ is an edge, contradicting the minimality of $i$. Now, since $v_{r}$ is not a leaf, there exists a minimal $k<r$ such that $\left\{u_{k}, v_{r}\right\}$ is an edge. Arguing as above, we have that $k=1$. Then $\left\{u_{1}, u_{r}\right\}$ and $\left\{u_{1}, v_{r}\right\}$ are both edges, and this contradicts the fact that $\left\{u_{r}, v_{r}\right\}$ is transversal.

Therefore, $\Delta$ is shellable by Theorem 4.5 , and it is well known that this implies that $\pi(G)$ is a Cohen-Macaulay graph (for instance, see the book of Bruns and Herzog [5, Theorem 5.1.13]).

For the following result, we recall that an ideal $I \subseteq S$ is connected in codimension 1 if any two minimal primes $\wp, \wp^{\prime}$ of $I$ are 1 -connected, i.e., there exists a path $\wp=\wp_{1}, \ldots, \wp_{m}=\wp^{\prime}$ of minimal primes of $I$ such that ht $\left(\wp_{i}+\wp_{i+1}\right)=\operatorname{ht}(I)+1$. If $I=I_{\Delta}$ is a square-free monomial ideal, then $I$ is connected in codimension 1 if and only if $\Delta$ is strongly connected, i.e., if and only if it is possible to walk from a facet to another passing through faces of codimension 1 in $\Delta$.

Theorem 4.7 Let $G$ be a graph satisfying the WSC and set $\Delta=\Delta(I(G))$ the simplicial complex associated to the edge ideal. The following conditions are equivalent:

(i) G has a unique perfect matching;

(ii) G has a unique perfect matching of transversal edges;

(iii) $\pi(G)=G$;

(iv) $\Delta$ is shellable;

(v) $G$ is Cohen-Macaulay;

(vi) $I(G)$ is connected in codimension 1 .

Proof (iii) $\Rightarrow$ (iv) is Theorem 4.6. (iv) $\Rightarrow$ (v) follows from [5, Theorem 5.1.13]. (v) $\Rightarrow$ (vi) is a general fact proved by Hartshorne in [10]. (iii) $\Rightarrow$ (ii) follows immediately from Lemma 4.4.

We want to show that (vi) $\Rightarrow$ (iii). Suppose that $\pi(G) \neq G$. This means that there is a bipartite complete subgraph of $G$, say $H$, with more than two vertices and such that any edge of $H$ is a transversal edge of $G$. Let $V(H)=A \cup B$ be the bipartition 
of the vertex set of $H$, and assume that $|A| \geq 2$. It is easy to construct a basic 1 -cover $\alpha$ that associates 1 to the vertices in $A$ and 0 to the vertices in $B$, and a basic 1 -cover $\beta$ that associates 0 to the vertices in $A$ and 1 to the ones in $B$. Consider the two ideals of $S$,

$$
\begin{aligned}
& \wp_{\alpha}=\left(x_{i}: \alpha(i)=1\right), \\
& \wp_{\beta}=\left(x_{i}: \beta(i)=1\right) .
\end{aligned}
$$

The ideals $\wp_{\alpha}$ and $\wp_{\beta}$ are minimal prime ideals of $I(G)$. We claim that they are not 1 -connected. If they were, then there would be a minimal prime ideal $\wp$ of $I(G)$ such that there exist $i, j \in A$ with $x_{i} \in \wp$ and $x_{j} \notin \wp$. Therefore, the basic 1-cover $\gamma$ associated to $\wp$ satisfies $\gamma(i)=1$ and $\gamma(j)=0$. As $H$ is a complete bipartite graph, each vertex of $A$ is connected to all vertices of $B$. So, because $\gamma$ is a 1-cover, it must also associate 1 to every vertex of $B$, and this contradicts the fact that $H$ consists of transversal edges.

Now we are going to show that (ii) $\Rightarrow$ (iii). If $G$ has a perfect matching of transversal edges, it is straightforward to check that it is unmixed. By [2, Theorem 2.8], the connected components of $G^{0-1}$ are all of the type $K_{r, r}$ for some $r \geq 1$. If $G$ were different from $\pi(G)$, then at least one of the $r$ 's would be greater than 1 . So we could find another perfect matching of transversal edges of $G$ by changing the matching of $K_{r, r}$ induced by the initial matching on $G$.

For the implication (i) $\Rightarrow$ (ii), let $M=\left\{\left\{a_{1}, b_{1}\right\}, \ldots,\left\{a_{m}, b_{m}\right\}\right\}$ be the unique perfect matching of $G$. Suppose that an edge in $M$, say $\left\{a_{1}, b_{1}\right\}$, is not transversal. Since $G$ satisfies the WSC, there is an $i>1$ such that $\left\{a_{1}, b_{i}\right\}$ (respectively $\left\{a_{1}, a_{i}\right\}$ ) is a transversal edge. But then $\left\{b_{1}, a_{i}\right\}$ (respectively $\left.\left\{b_{1}, b_{i}\right\}\right)$ is an edge by the weak square condition. So $M^{\prime}=\left\{\left\{a_{1}, b_{i}\right\},\left\{a_{2}, b_{2}\right\}, \ldots,\left\{a_{i}, b_{1}\right\}, \ldots,\left\{a_{m}, b_{m}\right\}\right\}$ (respectively $\left.M^{\prime}=\left\{\left\{a_{1}, a_{i}\right\},\left\{a_{2}, b_{2}\right\}, \ldots,\left\{b_{i}, b_{1}\right\}, \ldots,\left\{a_{m}, b_{m}\right\}\right\}\right)$ is another matching, a contradiction.

It remains to show that (ii) $\Rightarrow$ (i). But we already proved that if (ii) holds, then $G$ is Cohen-Macaulay. In particular $G$ is unmixed, so any other perfect matching of $G$ is forced to consist of transversal edges.

Whereas graphs whose edge ideal has a linear resolution have been completely characterized by Fröberg in [8], it is still an open problem (even in the bipartite case) to characterize in a combinatorial fashion the Castelnuovo-Mumford regularity of the edge ideal. A general result in [16] asserts that a lower bound for $\operatorname{reg}(S / I(G))$ is the maximum size of a pairwise disconnected set of edges of $G$. Moreover, by the present paper it easily follows that the ordered matching number of $G$ provides an upper bound for $\operatorname{reg}(S / I(G)$ ) (see the remark below). In [26], Zheng showed that if $G$ is a tree, then $\operatorname{reg}(S / I(G))$ is actually equal to the maximum number of disconnected edges of $G$. Later, in [9], Hà and Van Tuyl showed that the same conclusion holds for chordal graphs, and recently, the author of [17] showed this equality in the bipartite unmixed case, too. As another application of the operator $\pi$, we show in Theorem 4.10 that this equality holds also for any bipartite graph satisfying the WSC, extending the result of Kummini. First notice that to prove his theorem, Kummini defined a new graph, called the acyclic reduction, starting from a bipartite unmixed graph [17, Discussion 2.8]. It is possible to show that this new graph coincides with 
$\pi(G)$. So in some sense, $\pi(G)$ can be seen as an extension to the class of all graphs of the acyclic reduction defined in [17].

Remark 4.8 We showed in Corollary 2.11 that, for any graph $G$, we have $\operatorname{ara}\left(J(G) S_{\mathfrak{m}}\right) \leq v_{o}(G)+1$, provided the field $K$ is infinite. Recall that the cohomological dimensions of $J(G) S_{\mathfrak{m}}$ and of $J(G)$ agree, i.e., $\operatorname{cd}\left(S_{\mathfrak{m}}, J(G) S_{\mathfrak{m}}\right)=$ $\operatorname{cd}(S, J(G))$. But by a result in [19] the cohomological dimension of $J(G)$ is equal to the projective dimension of $S / J(G)$. Since the cohomological dimension is a lower bound for the arithmetical rank, we have that $\operatorname{pd}(S / J(G)) \leq v_{o}(G)+1$. As $I(G)$ is the Alexander dual of $J(G)$, it follows by [24] that

$$
\operatorname{reg}(S / I(G)) \leq v_{o}(G)
$$

The above inequality holds also if $K$ is finite, since the extension of scalars from $S / I(G)$ to $S / I(G) \otimes_{K} \bar{K}$, where $\bar{K}$ is the algebraic closure of $K$, does not change the regularity. Since $v_{o}(G)$ is less than or equal to the matching number of $G$ by definition, the above inequality strengthens [9, Theorem 1.5].

Lemma 4.9 Let $G$ be any graph. Then

$$
\operatorname{reg}(S / I(G))=\operatorname{reg}\left(S^{\prime} / I(\pi(G))\right),
$$

where $S^{\prime}=K\left[y_{1}, \ldots, y_{p}\right]$ is the polynomial ring in $p=|V(\pi(G))|$ variables over $K$.

Proof For any $i=1, \ldots, p$, call $V_{i}$ the set of vertices of $G$ that collapses to the vertex $i$ of $\pi(G)$. Then consider the homomorphism

$$
\begin{aligned}
\phi: S^{\prime} & \longrightarrow S, \\
y_{i} & \mapsto \prod_{j \in V_{i}} x_{j}=: m_{i} .
\end{aligned}
$$

By the correspondence of basic 1-covers of $G$ and $\pi(G)$ described in Proposition 4.2, one easily sees that $\phi(J(\pi(G))) S=J(G)$. Moreover, it is obvious that $m_{1}, \ldots, m_{p}$ form a regular sequence of $S$, so by a theorem of Hartshorne ([11, Proposition 1]) $S$ is a flat $S^{\prime}$-module via $\phi$. Then if $F_{\bullet}$ is a minimal free resolution of $S^{\prime} / J(\pi(G))$ over $S^{\prime}$, it follows that $F_{\bullet} \otimes \otimes_{S^{\prime}} S$ is a minimal free resolution of $S / J(G)$ over $S$. Therefore, the total Betti numbers of $S^{\prime} / J(\pi(G))$ and of $S / J(G)$ are the same, and in particular $\operatorname{pd}(S / J(G))=\operatorname{pd}\left(S^{\prime} / J(\pi(G))\right)$. Thus [24] yields the conclusion.

Theorem 4.10 Let $G$ be a bipartite graph satisfying the WSC. Then the CastelnuovoMumford regularity of $S / I(G)$ is equal to the maximum size of a pairwise disconnected set of edges of $G$.

Proof By Lemma 4.9, using the same notation, $\operatorname{reg}(S / I(G))=\operatorname{reg}\left(S^{\prime} / I(\pi(G))\right)$. Moreover, the maximum size of a pairwise disconnected set of edges in $G$ is equal to the same number for $\pi(G)$. Since $\pi(G)$ is Cohen-Macaulay by Theorem 4.6, one can deduce the conclusion using [12, Corollary 2.2.b]. 
Acknowledgements The authors wish to thank Jürgen Herzog for suggesting this topic and for many useful discussions which led to new stimulating questions and interesting observations. We also wish to thank Aldo Conca and Bruno Benedetti for their useful comments.

\section{References}

1. Benedetti, B., Constantinescu, A., Varbaro, M.: Dimension, depth and zero-divisors of the algebra of basic $k$-covers of a graph. Le Matematiche LXIII(II), 117-156 (2008)

2. Benedetti, B., Varbaro, M.: Unmixed graphs that are domains. Commun. Algebra (2009, to appear)

3. Birkhoff, G.: Lattice Theory, 3rd edn. Am. Math. Soc. Colloq. Publ., vol. 25. Am. Math. Soc., Providence (1967)

4. Björner, A.: Shellable and Cohen-Macaulay partially ordered sets. Trans. Am. Math. Soc. 260, 159183 (1980)

5. Bruns, W., Herzog, J.: Cohen-Macaulay Rings. Cambridge Studies in Advanced Mathematics (1993)

6. Bruns, W., Vetter, U.: Determinantal Rings. Lecture Notes in Mathematics, vol. 1327 (1980)

7. De Concini, C., Eisenbud, D., Procesi, C.: Hodge algebras. Astérisque, 91 (1982)

8. Fröberg, R.: On Stanley-Reisner Rings. Topics in Algebra, Banach Center Publications, Part 2, vol. 26, pp. 57-70. Springer, Berlin (1990)

9. Hà, H.T., Van Tuyl, A.: Monomial ideals, edge ideals of Hypergraphs, and their graded Betti numbers. J. Algebr. Comb. 27(2), 215-245 (2008)

10. Hartshorne, R.: Complete intersection and connectedness. Am. J. Math. 84, 497-508 (1962)

11. Hartshorne, R.: A property of $A$-sequences. Bull. Soc. Math. Fr. 94, 61-65 (1966)

12. Herzog, J., Hibi, T.: Distributive lattices, bipartite graphs and Alexander duality. J. Algebr. Comb. 22(3), 289-302 (2005)

13. Herzog, J., Hibi, T., Trung, N.V.: Symbolic powers of monomial ideals and vertex cover algebras. Adv. Math. 210, 304-322 (2007)

14. Hibi, T.: Distributive lattice, affine semigroup rings and algebras with straightening laws. Adv. Stud. Pure Math., 11 (1987)

15. Kalkbrener, M., Sturmfels, B.: Initial complex of prime ideals. Adv. Math. 116, 365-376 (1995)

16. Katzman, M.: Characteristic-independence of Betti numbers of graph ideals. J. Comb. Theory, Ser. A 113(3), 435-454 (2006)

17. Kummini, M.: Regularity, depth and arithmetic rank of bipartite edge ideals. J. Algebr. Comb. 30(4), 429-445 (2009)

18. Lovász, L., Plummer, M.D.: Matching Theory. North-Holland Mathematics Studies, vol. 121. Annals of Discrete Mathematics, vol. 29. North-Holland, Amsterdam (1986). Akadémiai Kiadó (Publishing House of the Hungarian Academy of Sciences), Budapest

19. Lyubeznik, G.: On the local cohomology modules $H_{\mathfrak{U}}^{i}(R)$ for ideals $\mathfrak{U}$ generated by an $R$-sequence. In: Complete Intersection. Lect. Notes in Math., vol. 1092, pp. 214-220. Springer, Berlin (1984)

20. Lyubeznik, G.: On the arithmetical rank of monomial ideals. J. Algebra 112(1), 86-89 (1988)

21. Morey, S., Reyes, E., Villarreal, R.H.: Cohen-Macaulay, shellable and unmixed clutters with a perfect matching of König type. J. Pure Appl. Algebra 212, 1770-1786 (2008)

22. Northcott, D.G., Rees, D.: Reduction of ideals in local rings. Proc. Camb. Philos. Soc. 50, 145-158 (1954)

23. Rinaldo, G.: Koszulness of vertex cover algebras of bipartite graphs. Commun. Algebra (2009, to appear)

24. Terai, N.: Alexander duality theorem and Stanley-Reisner rings. Free resolutions of projective varieties and related topics. Sūrikaisekikenkyūsho Kōkyūroku 1078, 174-184 (1999)

25. Varbaro, M.: Gröbner deformations, connectedness and cohomological dimension. J. Algebra 322, 2492-2507 (2009)

26. Zheng, X.: Resolution of facet ideals. Commun. Algebra 32(6), 2301-2324 (2004) 